\title{
Clinical Awareness and a Postulated Pathway for Testicular Tumors Forming in an Obese Society with Simultaneous Legalization of Cannabinoid's. Case Report and Literature Review
}

\author{
Ahmed M El Farrah ${ }^{*}$ \\ Palm Springs Hospital, Palm Springs, Hospital in Miami, Florida, USA \\ *Corresponding author: Ahmed M El Farrah, Palm Springs Hospital, Palm Springs, Hospital in Miami, \\ Florida, USA, Tel: +305 558-2500 (ext. 4719); E-mail: amelfa256@gmail.com
}

Received Date: 20 October, 2017; Accepted Date: 13 April, 2018; Published Date: 27 June, 2018

\begin{abstract}
Current physiology in the body uses endogenous cannabinoids to do specific functions. Recently there has been evidence to show that there is alteration of cannabinoid receptor sites with use of exogenous cannabinoid (marijuana) use. Exogenous cannabinoid use has been linked to some detrimental effects including depressed CNS activity, increased lipogenesis, and the formation of testicular masses with potentially fatal outcomes. This article presents a 33 y/o male who lived a sedentary life style and used cannabinoids casually and presented with a painful testicular mass. This article is the first to postulate a pathway in obese patients using exogenous cannabinoids; and its effects causing testicular masses. Also, this article hopefully brings awareness to clinicians concerning the effects of exogenous cannabinoids utilizing endogenous cannabinoid pathways and emphasizes the importance of thorough H/P's; and social histories in a society rapidly legalizing cannabinoid use.
\end{abstract}

\section{Keywords}

Exogenous cannabinoid; Non-Seminomatous tumors; Seminomatous tumors; Testicular germ cell tumors

\section{Abbreviations}

AEA: Anandamide or $\mathrm{N}$-arachidonoylethanolamine

CB1: Cannabinoid 1 receptor

CB2: Cannabinoid 2 receptor
DHEA-S: Dehydroepiandrosterone Sulfate

FAAH: Fatty acid amide hydrolase

FSH: Follicle-Stimulating Hormone

LH: Luteinizing Hormone

SHBG: Sex Hormone Binding Globulin

THC: Tetrahydrocannabinol

TNF-alpha: Tumor Necrosis Factor alpha

TRPV: Transient Receptor Potential Cation Channels, Vanilloid

2-AG: 2-Arachidonoylglycerol

\section{Introduction}

Testicular germ cell tumors (TGCT) are the most common type of malignancy in American men between 15 and 34 years of age. The age specific incidence of seminomas peaks around 35-40 years and non-seminomas about 10 years earlier at age 25-35. The risk factors TGCT both seminomatous and non-seminomatous types include an undescended testicle (cryptochordism), abnormal testicle development, genetic predisposition for testicular cancer within their family, age, and race. During the last half of the twentieth century, the incidence of testicular germ cell tumors has increased 3-6\% in the United States [1,2] With the prevalence of TGCT's high and increasing over the last few years, other aspects of American society (obesity, sedentary lifestyles) are also rapidly increasing. The correlation between cannabis use 
Citation: El Farrah AM (2018) Clinical Awareness and a Postulated Pathway for Testicular Tumors Forming in an Obese Society with Simultaneous Legalization of Cannabinoid's. Case Report and Literature Review. J Clin Case Rep Case Stud 2018: 14-18. DOI : https://doi.org/10.29199/2637-9309/CCCS-101014.

and the increased incidence of TGCT is already known; the exact mechanism is poorly understood, thus warranting further investigation [1]. This article postulates a mechanism using a pathway of what we already know thus far; and hypothesizes future outcomes as clinical data comes in. Co-incidentally, more than twothirds of Americans are either overweight or obese, due in part to readily available poor food choices, non-compliance in managed patients, and increasingly sedentary lifestyles, according to new estimates. Overweight and obesity are associated with a variety of chronic health conditions, which could potentially be avoided by preventing weight gain and obesity [2]. Lifestyle choices and recreational activities, such as the progressive expansion to legalize marijuana and its medicinal usages, are also becoming a rapid trend in American society along with a rapidly increasing obese population. Currently in 2017, with the most recent being Nevada, 26 states and the District of Columbia currently have laws broadly legalizing marijuana in some form. Seven of these states allow marijuana for recreational use. Arkansas, Florida, North Dakota and other states have approved exogenous cannabinoid's for medicinal use. With obesity and marijuana usage rapidly becoming part of society and culture, these two although pleasurable short-term, can have detrimental, if not deadly outcomes long term.

\section{Case presentation}

A 33-year-old white male was referred to Urology by his Primary Care Physician for a testicular cyst with newly associated pain. He had a past medical history significant for hypertension, obesity, and arthritis, which were stable. He had a past social history significant for daily alcohol consumption and casual marijuana usage since being a teenager, at least one time a week. He also made poor diet choices which gave him a BMI of 37. Upon examination by Urology, he stated he had been having pain ranging from 2-7 out of 10 for the last week. On physical exam, there was pain to palpation. He constantly directed attention to his right scrotum, were he complained of a lump or mass at the testicle. $\mathrm{He}$ stated; he noticed the mass for several months, but what raised his suspicion to follow up the lump was a newly acquired pain, tenderness, and swelling that came about randomly within the past week. At that time, he stated that there was no history of trauma; prior to the lump. Due to the onset of newly acquired pain with a testicular mass, exploratory surgery was warranted. He was counseled that an orchiectomy was a possibility. The initial work-up included ultrasound and a urinalysis that was sent for a urine culture. The ultrasound showed an atrophic right testicle; and a mass superior to the testicle. During the exploratory procedure, the mass was visualized encompassing the atrophic testicle and spermatochord, therefore; a right radical orchiectomy was warranted. An atrophic testicle with an enlarged mass attached was removed and sent for pathological examination and results (Figure 1). Pathology reported the mass being of mixed cell tissue with $70 \%$ identified as seminoma, and; the remaining $30 \%$ being of yolk sac tissue type. The seminoma consisted of polygonal cells forming 'sheet like' patterns interrupted by fibrous septa containing lymphocytes. The yolk sac tumor was diagnosed by its glandular and 'sinus like' pattern.

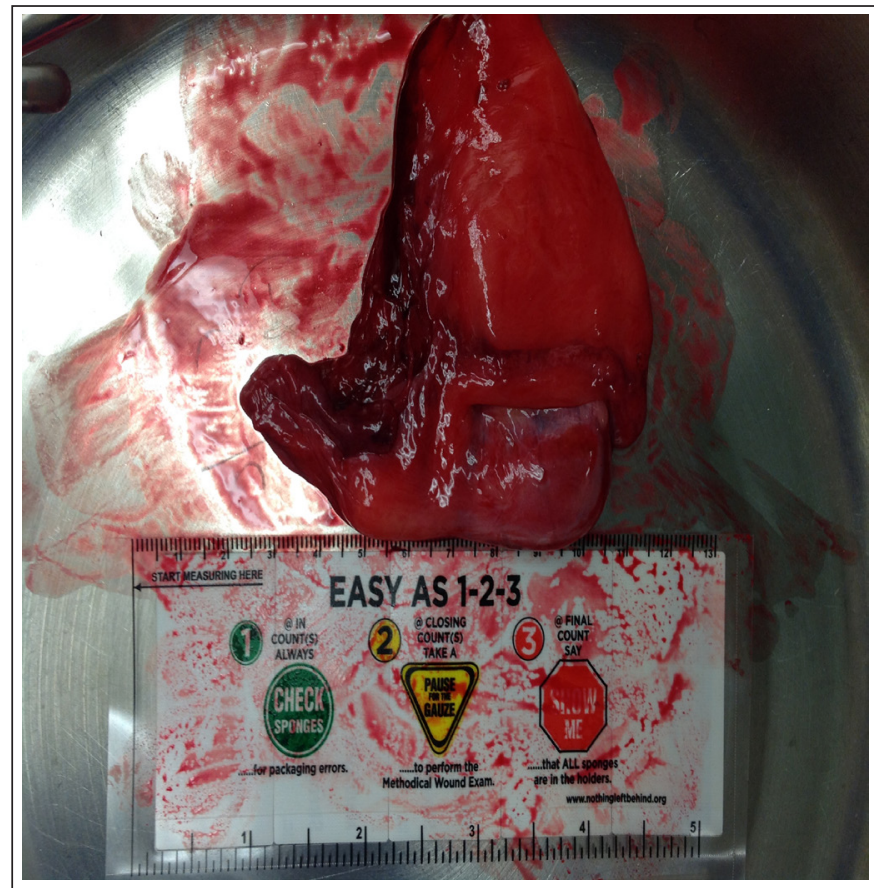

Figure 1: Seminomatous tissue displaying membranous immune positivity for CD117 and negativity for pan $-\mathrm{CK}$.

It has been shown that CD117 and pan CK are good tumor markers to diagnose seminomas tissue types, CD19 tumor markers are very sensitive for yolk sac tissue types, and CD30 markers are good to distinguish embryonal tissue types. The immunohistochemistry had CD117, -CK, and CD30, which supported the diagnosis. The seminoma showed membranous immune positivity for CD117 and negativity for pan -CK (Figure 2). The yolk sac showed immunopositivity for CD19, pan CK, and negativity for CD117 (Figure 3). The CD30 marker was negative in both tumors thus ruling out an embryonal tissue type. Pathology's final assessment was that the immunohistologic controls were performed and reacted appropriately. The mass attached to the testicle measured $2.6 \mathrm{~cm}$; and was limited to the testes without penetration through the tunica albicans. At that time, there was no lymphovascular invasion or penetration noted so it was staged as a $1 \mathrm{~A}$. Stage $1 \mathrm{~A}$ seminomatous tumors have a recurrence of metastasis to lymph nodes or other organs of in about $15 \%$ to $20 \%$ of cases, therefore; close surveillance with CT exams every 4-6 months was warranted. Due to the pathological diagnosis of a mixed seminoma and yolk sac tumor, hormonal tumor markers levels for AFP, LDH, and b-hCG were not warranted until the six months follow up provided that imaging studies ruled out metastasis. At his sixmonth follow-up for metastasis, a metastatic paracaval lymphnode was located. Although he started initial treatments of chemotherapy and radiation, the already metastasized tumor ultimately led to his demise three months later. 


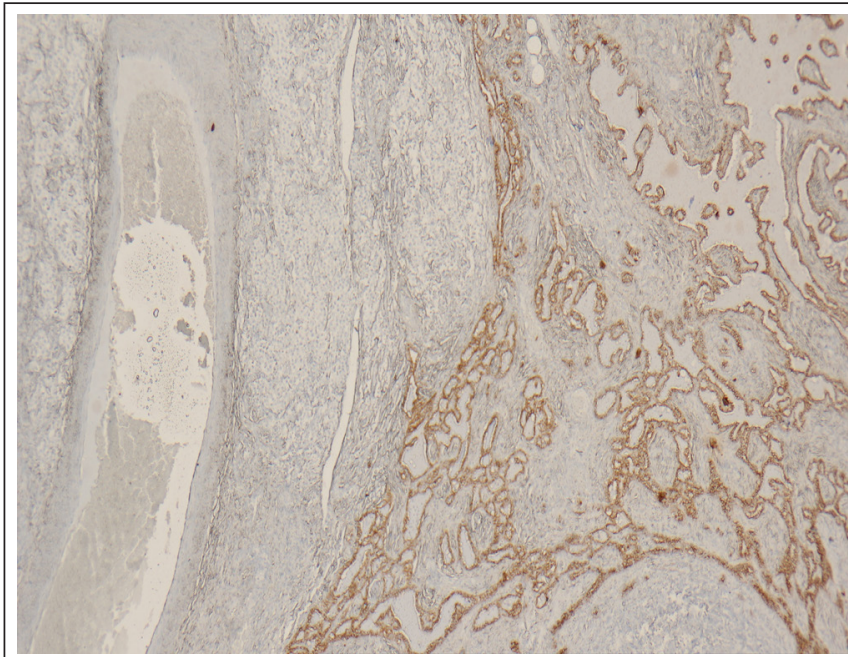

Figure 2: Yolk sac tissue displaying immunopositivity for CD19, pan CK, and negativity for CD117.

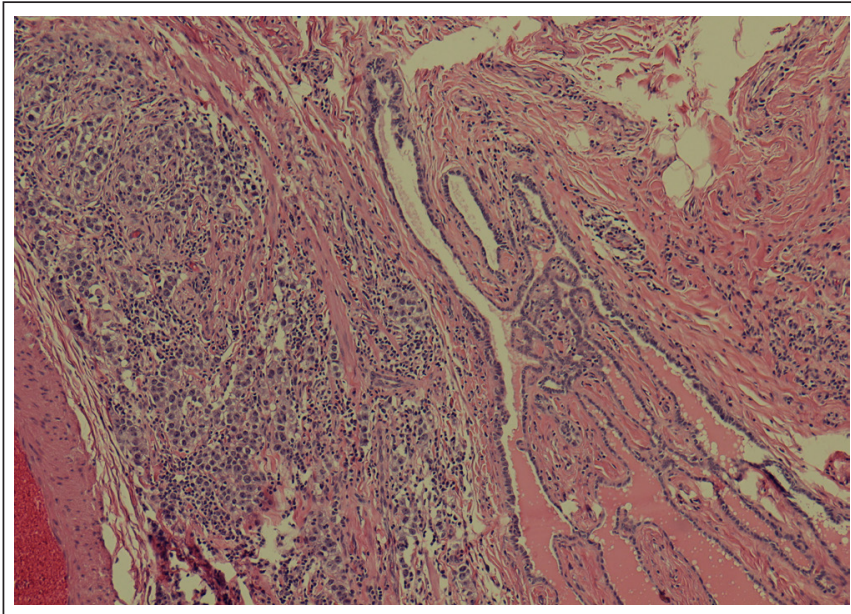

Figure 3: The atrophic testicle, spermatochord, and $2.6 \mathrm{~cm}$ mass limited to the testes without penetration through the tunica albicans.

\section{Discussion}

Although exogenous cannabinoids are transported to the liver and cleared via p450, they do not absorb in lipophilic tissues or in fat. They sit readily on lipid bi-layers for quicker access for adjacent organs. Exogenous cannabinoids can either block some cannabinoid receptor sites by blunting target organ activity, or; they can alter the normal function of pathophysiologic pathways used by endogenous cannabinoids. These pathways, when altered or blocked, cause the naturally occurring endogenous cannabinoids to linger in the periphery and be degraded into fatty acids starting lipogenesis in the liver $[3,4]$. In an obese patient, an already forged androgen pathway from hepatic lipogenesis is present (Figure 4). Lipogenesis occurring in this pathway will encourage production of carrier proteins such as SHBG and albumin for the transport of proteins. Androgens derived from cholesterol are in the form of testosterone and estradiol that are increased in the obese patient. The cholesterol feeding into lipogenesis is synthesized towards estradiol and not testosterone due to the mechanism of the carrier proteins. The carrier protein SHBG binds to free testosterone and albumin binds to increased and readily available androgens from cholesterol, forming estradiol. Estradiol in excess not only has a negative feedback inhibition to the HPO axis but also blunts protective immunological factors like TNF-alpha. This now forged androgen pathway is formed with bound up testosterone and increased levels of estradiol in the obese patient $[4,5]$. As previously stated, cannabinoids clear by way of p450 in the liver. In a specific example of altering pathophysiologic functioning, use of exogenous cannabinoids stimulate the endogenous cannabinoid receptors on the surface of lipid bi-layers; that have transient receptor potential cation channels (TRPV's). These receptor channels are highly calcium selective and located mostly on plasma membranes of numerous human and animal cell types [6]. TRPV's also have neural desensitization affects; causing blunting of normal neurophysiology. The exogenous cannabinoids, which are not stored away in fat like any basic drug going to the liver does, sit readily available for TRPV's located on the lipid bi-layers of plasma membranes [7]. The exogenous cannabinoids 'casually' disrupt endogenous cannabinoid physiology at these sites allowing endogenous cannabinoids, anamide and 2-arachidonoylglycerol (i.e. AEA, 2-AG); to be in excess in the peripheral circulation [8]. These peripheral endogenous cannabinoids now accumulated in excess, are then degraded at target organs by FAAH (with a short half-life) increasing fatty acids. This process; stimulates and feeds into lipogenesis, and if the individual is obese, this process will also feed into their forged androgen pathway $[3,5]$. As previously stated, in regards to the obese patient, the cholesterol from the increased lipogenesis is converted to testosterone and estradiol with inappropriate trends toward estradiol. The negative feedback mechanism to the HPO axis stops $\mathrm{GnRH}$ release while exogenous cannabinoids block receptor binding sites on the hypothalamus and pituitary gland. In the hypothalamus the satiety center is depressed causing hunger cravings, and at the pituitary gland, exogenous cannabinoids directly inhibit LH release needed for testicular maturation and spermatogenesis [8]. Simultaneously, exogenous cannabinoids bind to TRPV receptor sites preventing endogenous cannabinoids from relaying proper testicular maturation signals to the testicle by way of calcium ligand channels. Because these channels are blocked, the testicle does not get its neuroendocrine signal for maturation $[8,9]$. The blunted neurological response to the testicle accompanied with a forged androgen pathway and degraded endogenous cannabinoids feeding into lipogenesis are now a deadly combination leading into the 'Two hit hypothesis' and a genetic predisposition for tumors.

Following the 'Two hit hypothesis', a gene mutates to causing a loss or reduction in its function. The cells can now decrease tumor surveillance and progress to cancer, usually in combination with other genetic changes. The loss of these genes may be important more than proto-oncogene/oncogene activation with forming testicular tumors as well as different kinds of human cancer cells [10]. With overactive androgen pathways in the 


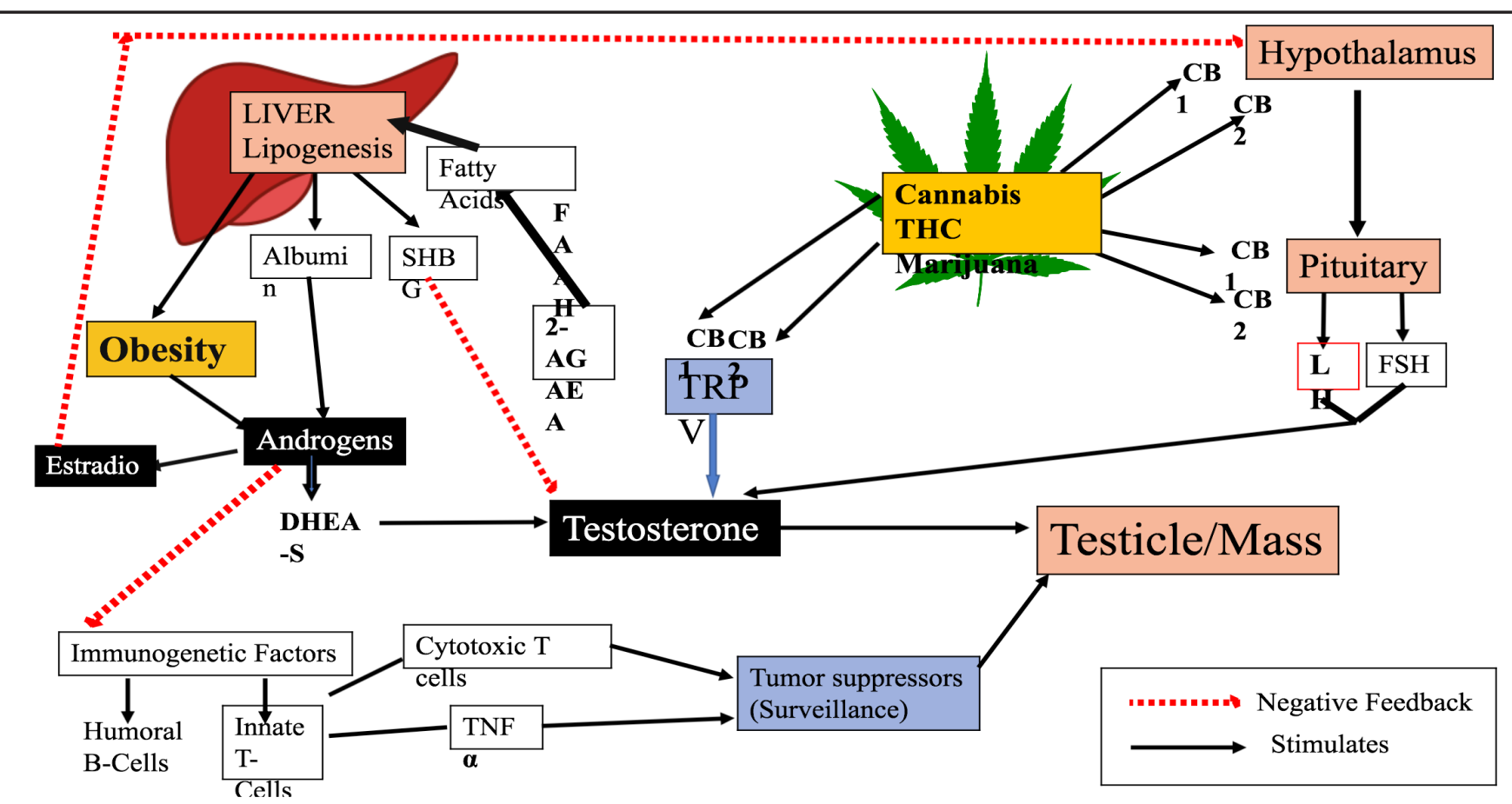

Figure 4: Postulated pathway connecting exogenous cannabinoid's in an already forged androgen pathway from obesity, and formation of a genetically predisposed seminomatous or non-seminomatous tumor.

1. Starting at the marijuana figure, exogenous cannabinoids blunt LH at the anterior pituitary, blunt affects of hormones at the hypothalamus, and blunt normal function of TRPV's for proper Testicular maturation.

2. At TRPV receptor exogenous cannabinoids block receptor sites allowing endogenous cannabinoids to stay in the periphery and be degraded in to fatty acids and feed into the lipogenesis pathway.

3. At the Liver, SHBG is released blunting testosterone synthesis and Androgens are formed

4. Androgen in the peripheral have a negative feedback mechanism to the hypothalamus and HPO axis and decrease immune surveillance.

5. In the immune system certain immunological factor are halted like TNF that decrease tumor surveillance which leave an individual susceptible to their applicable genetic predisposition of a testicular mass.

obese already being fed by their sedentary lifestyles, this postulated pathway further feeds into the programmed androgen pathway, thus decreasing immune and tumor surveillance making them susceptible to genetic predispositions for non-seminomatous and seminomatous tumors. This allows for the potential of a mass to form instead of the natural pathophysiology process into spermatogenesis and testicular maturation [8]. The combination of the factors, as previously stated, divert testosterone synthesis towards an established forged androgen pathway that ultimately decreases immune and tumor surveillance, leaving an individual susceptible to their genetic predispositions of a seminomatous or non-seminomatous tumors [8]. The proposed pathway divides people into four different categories based on obesity and cannabinoid use: 1) obese people who do not use exogenous cannabinoids (obese non-users), 2) obese people who do use exogenous cannabinoids (obese users), 3) lean people who use exogenous cannabinoids (non-obese users), and 4) lean people who do not use exogenous cannabinoids (non-obese non-users). In the obese-non-user, these individuals do not have blunting of endogenous cannabinoid receptors along the HPO axis that will depress the satiety center at the hypothalamus and blunt $\mathrm{LH}$ release at the pituitary. These individuals do have the HPO axis blunted by excess estradiol from their forged androgen pathway, but the TRPV's and neuroendocrine pathways are intact for some spermatogenesis and testicular maturation. In the non-obese users, these individuals do not have the forged androgen pathway producing estradiol from cholesterol that has the negative feedback mechanism to the HPO axis. These individuals have excess endogenous cannabinoids being degraded by FAAH and go into lipogenesis, however; the lipogenesis in these does not fuel a forged androgen pathway. In the obese user, these individuals have the commonality of the endogenous cannabinoids in the periphery being degraded by FAAH feeding into lipogenesis, as well as their already forged androgen pathway 
Citation: El Farrah AM (2018) Clinical Awareness and a Postulated Pathway for Testicular Tumors Forming in an Obese Society with Simultaneous Legalization of Cannabinoid's. Case Report and Literature Review. J Clin Case Rep Case Stud 2018: 14-18.

with estradiol synthesized from cholesterol. The obese user also has exogenous cannabinoids blocking receptor sites from proper endogenous cannabinoid function. The final population of nonobese non-users has none of the aforementioned deleterious effects and can be used as a control group in future studies to verify if the postulated pathway exposes obese cannabinoid users to genetic predispositions for the formation of a testicular mass and if this manifests clinically.

\section{Conclusion}

This article postulates a pathway bringing together previously discovered scientific and clinical data, in obese patients, with coinciding use of exogenous cannabinoids and the case presentation portraying a possible trend in the near future. Further investigation of TRPV's and lipogenesis pathways are warranted to solidify the postulated pathway. (1) Investigation is also warranted for the postulated detrimental effects that ultimately decrease immune and tumor surveillance, leaving a male individual susceptible to his genetic predisposition for producing a seminomatous/non-seminomatus mass instead of healthy testicular tissue by way of spermatogenesis in the HPO pathway. This article also hopes to bring attention that around the time the first articles in 2012 were published, 2 states legalized recreational exogenous cannabinoids use and 11 for medicinal use. (2) Currently in 2017, with the most recent being Nevada, 8 states have legalized cannabinoids, and another 19 states approve exogenous cannabinoid's for medicinal use. (3) Finally, this article hopes to remind clinicians of the importance of thorough histories, including social activities, and complete physical exams including appropriate screening for testicular masses as society rapidly approaches obesity and legalized cannabinoids.

\section{Consent}

Written informed consent was obtained from the patient for publication of this case report and any accompanying images.

\section{Conflict of Interest}

The authors declared no potential conflict of interests with respect to the research authorship, and/or publication of this paper. The work on the present paper was not funded by any source.

\section{References}

1. Lacson JC, Carroll JD, Tuazon E, Castelao EJ, Bernstein L, et al. (2012) Population-Based Case-Control Study of Recreational Drug Use and Testis Cancer Risk Confirms Association between Marijuana Use and Non-Seminoma Risk. Cancer 118: 5374-5383.

2. Looijenga LH, Oosterhuis JW (1999) Pathogenesis of testicular germ cell tumours. Rev Reprod 4: 90-100.

3. Osei-Hyiaman D, DePetrillo M, Pacher P, Liu J, Radaeva S, et al. (2005) Endocannabinoid activation at hepatic CB1 receptors stimulates fatty acid synthesis and contributes to diet-induced obesity. J Clin Invest 115: 1298-1305.

4. Selva DM, Hogeveen KN, Innis SM, Hammond GL (2007) Monosaccharide-induced lipogenesis regulates the human hepatic sex hormone-binding globulin gene. J Clin Invest 117: 3979-3987.

5. Simó R, Barbosa-Desongles A, Hernandez C, Selva DM (2012) IL1 $\beta$ down-regulation of sex hormone-binding globulin production by decreasing HNF-4 $\alpha$ via MEK-1/2 and JNK MAPK pathways. Mol Endocrinol 26: 1917-1927.

6. Grotenhermen F, Müller-Vahl K (2012) The therapeutic potential of cannabis and cannabinoids. Dtsch Arztebl Int 109: 495-501.

7. Simó R, Barbosa-Desongles A, Lecube A, Hernandez C, Selva DM (2012) Potential role of tumor necrosis factor-alpha in downregulating sex hormone-binding globulin. Diabetes 61: 372-382.

8. Aizpurua-Olaizola O, Elezgarai I, Rico-Barrio I, Zarandona I, Etxebarria N, et al. (2017) Targeting the endocannabinoid system: future therapeutic strategies. Drug Discov Today 22: 105-110.

9. Pagotto U, Marsicano G, Cota D, Lutz B, Pasquali R (2006) The emerging role of the endocannabinoid system in endocrine regulation and energy balance. Endocr Rev 27: 73-100.

10. Weinberg, Robert A (2014) The Biology of Cancer. Garland Science, page 231 . 